Eur. J. Clin. Chem. Clin. Biochem.

Vol. 29, 1991, pp. $481-485$

(C) 1991 Walter de Gruyter \& Co.

Berlin - New York

\title{
A Cationic Glycoprotein Pattern in Human Serum and Cerebrospinal Fluid with Pathological Implications ${ }^{1}$ )
}

\author{
By M. Mäder, K. Retzlaff and K. Felgenhauer \\ Neurologische Klinik der Universität Göttingen
}

(Received December 20, 1990/May 8, 1991)

\begin{abstract}
Summary: Patterns of highly glycosylated proteins with mainly cationic isoelectric points, $\mathrm{pH} 6.5-9.5$, were observed in serum and cerebrospinal fluid of patients with various disorders. Detection was performed after isoelectric focusing, using an immunoassay specific for digoxigenylated carbohydrate moieties of glycoconjugates. To our knowledge, these glycoproteins have not hitherto been described as regular serum proteins. The patterns were found among $7 \%$ of the patients studied $(n=400)$. Similar bands were not detectable in a reference group of 100 persons without clinical symptoms. The glycoprotein pattern was specific for each individual. The pathophysiological meaning of these glycoproteins as well as the basic biochemistry has not yet been evaluated. The glycoproteins, however, were shown to differ from immunoglobulin $G$, oligoclonal immunoglobulin $\mathrm{G}$, paraprotein or from regular cationic serum protein. By comparison with standard glycoproteins a carbohydrate content of $30 \pm 10 \%$ was roughly suggested. The oligosaccharides contain probably sialic acid as evidenced by lectin binding. Although the diagnoses varied, $90 \%$ of the patients with this glycoprotein pattern had inflammatory processes.
\end{abstract}

\section{Introduction}

Oligoclonal immunoglobulin $\mathrm{G}$ patterns of cerebrospinal fluid proteins separated by isoelectric focusing and stained with Coomassie blue or silver are sensitive markers of inflammatory processes in the brain (1). During an investigation of these patterns in 20 oligoclonal positive cerebrospinal fluids using Western blots and an immunoassay selective for carböhydrate moieties, we discovered a distinct glycoprotein pattern in one sample (No. 12 in fig. 2). This pattern was also found in the corresponding serum. Although a diagnosis wạs initially nọt possible for that patient, half a year later high titres of autoantibodies were found. Similar glycoprotein patterns were also detected in the sera of other patients suffering from various diseases.

') Supported by the Deutsche Forschungsgemeinschaft, SFB 330 , B6.
The aim of this first study was to elucidate the relationship of the glycoprotein pattern

i) to pathological processes and

ii) to know serum components found in the cationic range in isoelectric focusing.

\section{Materials and Methods}

All samples studied were obtained from the diagnostic laboratories of the clinic: 20 paired cerebrospinal fluid and serum samples with oligoclonal immunoglobulin $G$ bands in the cerebrospinal fluid, 300 consecutive serum samples of patients with various neurological complaints and 100 consecutive serum samples of patients with various internal diseases. One hundred reference samples from persons without clinical symptoms were taken from the blood bank.

Protein was determined according to 1.c. (2). Sodium dodecylsulphate gel electrophoresis and Coomassie blue staining werc performed according to 1.c. (3). The method of 1.c. (4) was used for the periodate acid Schiffs stain. Western blots after sodium dodecylsulphate gel electrophoresis were performed according to 1.c. (5). Western blots after isoelectric focusing werc carried out according to $1 . c$. (6) using pressure-diffusion blotting. 
Isoelectric focusing and Coomassie blue staining of these gcls were performed as described by 1.c. (7). Briefly: gels were prepared exactly as described by $A$. Winter et al. 1977 in the Application Note 250, LK B-Produkter, Bromma Sweden. The final gel mix contained $40 \mathrm{~g} / 1$ urea, $48.5 \mathrm{~g} / 1$ acrylamide, $1.5 \mathrm{~g} / \mathrm{l}$ bisacrylamide, $50 \mathrm{ml} / \mathrm{l}$ ampholine $1: 1 \mathrm{LKB} / \mathrm{Pharmalyte}, 0.15$ $\mathrm{g} / 1$ tetramethyl-ethylenediamine, $0.3 \mathrm{~g} / 1$ ammonium peroxidisulphate.

For glycan detection the method of 1.c. (8) was used. Briefly: the hydroxyl groups of the carbohydrate moieties of glycoconjugates were oxidized by periodic acid to aldehyde groups, which then reacted with digoxigenin-succinyl- $\varepsilon$-amido caproic acid hydrazide. A high affinity antibody, conjugated to alkaline phosphatase, was used for the detection of the incorporated digoxigenin. Oxidation of carbohydrates and coupling of digoxigenin can be performed before separation of proteins on gels (method A, which is more sensitive), or these stages can be performed after blotting, directly on the blots (method B, which is less sensitive).

Lectin detection (9) was carried out as follows: 6 lectins of different specificity (see below) conjugated with digoxigenin were employed. The detection procedure was the same as described above for the glycan detection assay. The lectins (tab. 2) were from Boehringer, Mannheim.

\section{Results}

Glycoprotein pattern of sera and cerebrospinal fluids

In figure 1 , a screening blot comprising regularly $23-$ 25 lanes is depicted. Various sera of neurological patients were separated by isoelectric focusing and after blotting probed by the glycan detection assay.
Three sera (Nos. 5, 16 and 21) show the typical glycan pattern. Three sera (Nos. 4, 22 and 23) show slightly developed patterns which were not deemed to be positive. This type of pattern is obtained only by method B (see Materials and Methods) since method A, though more sensitive, impairs isoelectric focusing seriously by oxidation and derivatization of the carbohydrates before separation.

The glycan pattern was found in $7 \%$ of all sera studied from patients with neurological $(n=300)$ and internal $(n=100)$ disorders. When paired cerebrospinal fluid and serum samples were investigated both showed identical patterns. One hundred sera of a reference group (blood bank samples) exhibited no glycan patterns (data not shown).

In figure 2, a collection of 21 glycan patterns out of 28 found in the 400 patients studied is depicted. The patterns range from $\mathrm{pH} 6.5-9.5$, vary from person to person, although some similarities can be recognized upon comparison.

In some blots, e. g. figure 1 , a few $(1-4)$ single more intensely stained bands are observed in the lower $\mathrm{pH}$ range $(5.7-7.3)$. These bands are absent on other blots, e. g. figures 2,3 . They do not correspond to the glycoprotein bands described here, which are mainly localized on the blots above those in the higher pHrange (figures 1,2 , and 3 ).

Tab. 1. Comparison of the lower detection limits of standard glycoproteins, after sodium dodecylsulphate gel electrophoresis and staining with Coomassie blue or periodic acid Schiffs on gels, or detection with the glycan detection assay on blots.

Group I, proteins with a carbohydrate content less than 10\%; group II, proteins with a carbohydrate content of $10 \%$ or higher (for details see Results).

Method A and B, two different methods for detecting glycoproteins on Western blots by the glycan detection assay (see Materials and Methods). -

\begin{tabular}{lllll} 
& $\begin{array}{l}\text { Coomassie blue } \\
\text { staining }\end{array}$ & $\begin{array}{l}\text { Periodic acid } \\
\text { Schiff's staining }\end{array}$ & Method A & Method B \\
\hline Group I & $1-5 \mu \mathrm{g}$ & $20-60 \mu \mathrm{g}$ & $0.1-1 \mu \mathrm{g}$ & $-\overline{1}$ \\
Group II & $1-5 \mu \mathrm{g}$ & $5-20 \mu \mathrm{g}$ & $0.01-0.1 \mu \mathrm{g}$ & $0.1-5 \mu \mathrm{g}$ \\
\hline
\end{tabular}

Tab. 2 Pattern formation of sera which exhibit a positive pattern with the glycan detection assay when probed with the lectins listed.

\begin{tabular}{llll}
\hline Lectin & & & Pattern \\
\hline $\begin{array}{l}\text { Abbre- } \\
\text { viation }\end{array}$ & Source & Specificity & different \\
\hline AAA & Anguilla anguilla & L-Fucose & Galactose $\beta(1-4) N$-acetyl-glucosamine \\
DSA & Datura stramonium & Mannose $\alpha(1-3), \alpha(1-6), \alpha(1-2)$ & + \\
GNA & Galanthus nivalis & Sialic acid $\alpha(2-3)$ galactose & + \\
MAA & Maackia amurensis & Galactose $\beta(1-3) N$-acetyl-galactosamine & + \\
PNA & Arachis hypogaea & Sialic acid $\alpha(2-6)$ galactose & + \\
SNA & Sambucus nigra & + & + \\
\hline
\end{tabular}




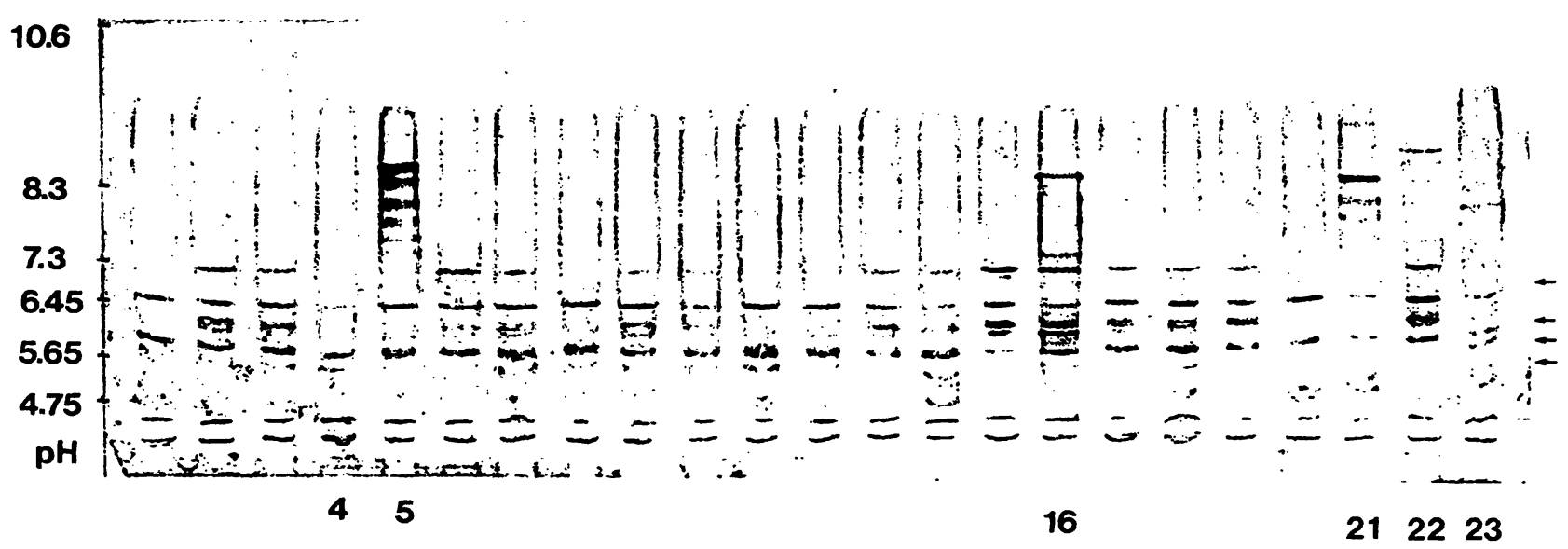

Fig. 1. Pressure-diffusion blot on nitrocellulose after isoelectric focusing and development by the glycan detection assay. The blot represents a screening unit comprising $23-25$ sera of consecutive patients. $5,16,21$, glycoprotein patterns. $4,22,23$, slightly stained patterns which were not deemed to be positive. The arrows indicate bands $(1-4 /$ lane) which do not belong to the glycoprotein pattern described. These bands are not prominent in all blots (compare the other figures).

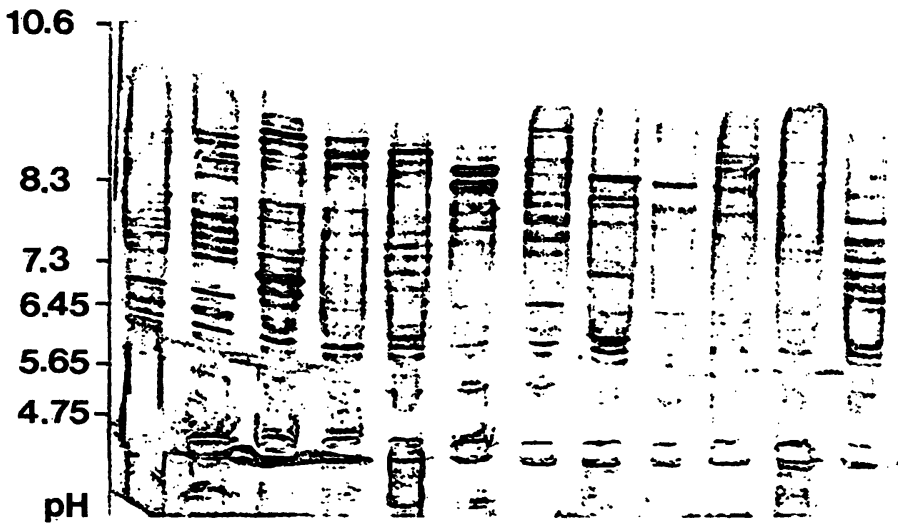

12

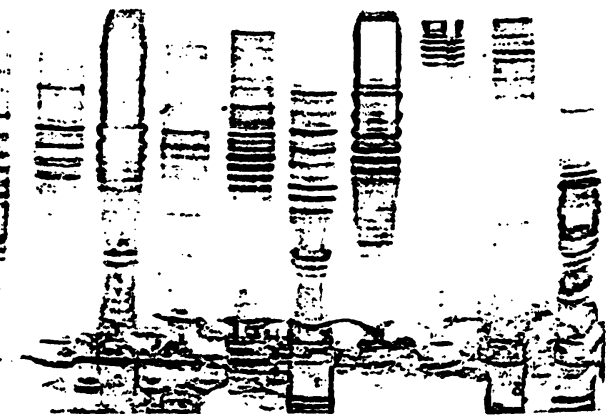

14

Fig. 2. Pressure-diffusion blot on nitrocellulose after isoelectric focusing of 21 sera of neurological patients, showing the glycoprotein pattern after development by the glycan detection assay. The patterns range from $\mathrm{pH} 6.5-9.5$. Similarities are observed among some patterns. 12, the pattern which was detected first, 14, the same patient as shown in figure 3 .

Lower detection limits of the glycan detection assay

To evaluate the minimal amounts necessary for glycan detection with the above assay, the following experiments were conducted. Several standard glycoproteins with various amounts of carbohydrates were stained with Coomassie blue or periodic acid Schiffs after separation by sodium dodecylsulphate polyacrylamide gel electrophoresis. Western blot analysis was performed by method A or B of the glycan detection assay (see Materials and Methods). The proteins under study were divided into two groups.

Group I, proteins with a carbohydrate content less than $10 \%$ :

ovalbumin $(1-2 \%)$,

immunoglobulin G (2-3\%),

transferrin $(6 \%)$,

coeruloplasmin $(7 \%)$,

immunoglobulin A (9\%).
Group II, proteins with a carbohydrate content of $10 \%$ or higher:

$\alpha_{2}$-macroglobulin (10\%),

haptoglobin (16\%),

horseradish peroxidase $(20 \%)$,

$\alpha_{1}$-acidic glycoprotein (40\%).

The results are summarized in table 1. $\alpha_{1}$-Acidic glycoprotein was the only protein which allowed detection by method $\mathrm{B}$ in amounts $(0.1 \mu \mathrm{g})$ lower than those necessary for Coomassie blue staining $(1 \mu \mathrm{g})$.

Biochemical nature of the glycoprotein pattern

As shown in figure 3, the glycan pattern on the Western blot is not identical to the Coomassie blue pattern in the polyacrylamide gel, although bands are occasionally observed which might correspond. Paraproteins do not stain with the glycan detection assay and 
a

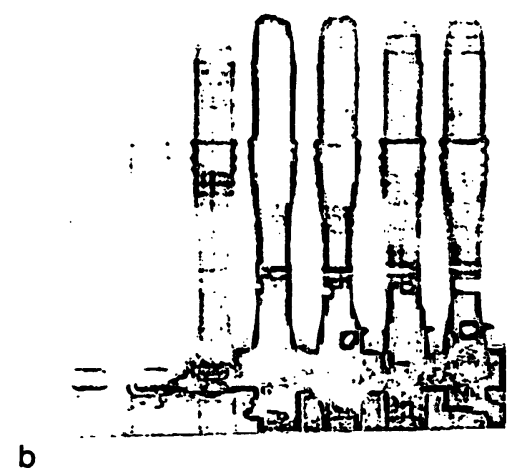

b

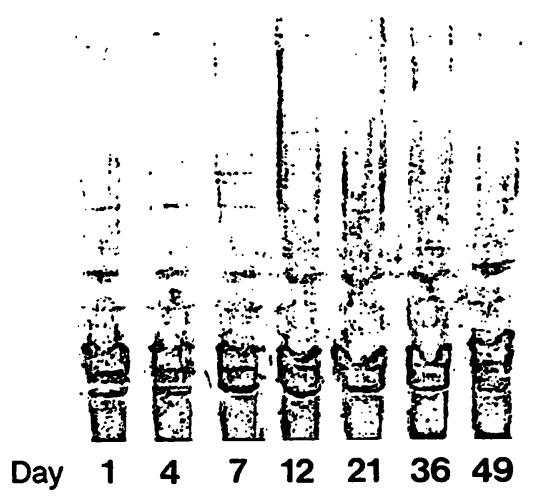

Fig. 3. a) Pressure-diffusion blot on nitrocellulose after isoelectic focusing of sera of one patient (Listeria meningitis) taken at the time points indicated (days) and developed by the glycan detection assay.

b) the corresponding Coomassie blue-stained protein patterns in the polyacrylamide gel after focusing.

oligoclonal immunoglobulin $\mathrm{G}$ does not react with the glycan detection assay, although, like paraproteins, it contains $2-3 \%$ carbohydrate (data not shown). The regular immunoglobulin $G$ pattern is totally different from the glycan pattern when detected by enzyme conjugated anti-immunoglobulin $\mathrm{G}$ after isoelectric focusing in the cationic range (data not shown).

Glycan pattern-positive sera were probed after focusing and blotting by a set of digoxigenin-conjugated lectins of various sugar-specificities. The results are summarized in table 2: some lectins cause a pattern very similar to the glycan pattern. Other lectins demonstrate a different pattern and some none whatsoever. The two lectins causing similar patterns were specific for sialic acid $\alpha(2-3)$ galactose and for galactose $\beta(1-3) N$-acetylgalactosamine, respectively.

Pathophysiological significance of the glycoconjugate pattern

The twenty-one neurological patients demonstrating the glycoprotein pattern in their serum had varying diagnoses. Nineteen, however, had inflammatory processes in the CNS as evidenced by elevated immunoglobulin levels, oligoclonal immunoglobulin or activated B-cells in the cerebrospinal fluid (1).

In figure 3, a longitudinal study of a patient with Listeria meningitis who died after 7 weeks is shown. Glycan and protein pattern (Coomassie blue stained) increase dramatically in the beginning and decrease slowly during the period of treatment. The pattern, however, does not change qualitatively.

\section{Discussion}

The glycoprotein pattern described here is obviously caused by pathophysiological processes as it only occurred in patients' sera. No specific disorder has been found as yet with a relation to these proteins. It seems likely that more basic processes, e.g. immunological reactions, have to be considered since most patients (90\%) showing the glycan pattern developed inflammatory processes.

We assume that the concentration of the glycoprotein in the samples applied ( $5 \mu$ l undiluted serum) lies just below the detection limit of Coomassie blue staining (probably $1 \mu \mathrm{g}$ ) whereas the more sensitive glycan detection yields positive results. When experiments were performed with standard glycoprotein a similar detection sensitivity was found for the $\alpha_{1}$-acid glycoprotein containing $40 \%$ carbohydrate. This protein demonstrated limits for glycan detection of $0.1-0.5$ $\mu \mathrm{g}$ and for Coomassie blue of $1 \mu \mathrm{g}$ in a sodium dodecylsulphate gel. Hence, a carbohydrate content of $30 \pm 10 \%$ is roughly suggested for the glycoprotein described here.

Lectin experiments confirm that the glycoprotein pattern is due to a single glycoprotein species. A specific lectin either shows the same pattern as the glycan detection assay with all sera studied, or it does not. Thus we assume that a highly glycosylated basic glycoprotein has been detected in the serum of patients, which might not have been described before, and which has been shown to be not identical to regular well known cationic serum proteins, e.g. immunoglobulins. There is some evidence that this protein also exists in variable amounts in healthy persons, but in amounts below the detection limit of the assay employed. In the literature (10), relatively few cationic proteins have been described in human serum which are not predominantly glycosylated. 
Gries and coworkers (11) have shown that acidic glycoproteins might shift to basic isoelectric points when treated with glycosidase. This possibility has to be considered as such processes might occur in the patients' blood. However, the glycoprotein pattern of the patient with severe Listeria meningitis (fig. 3) shows a variation in the quantity but not in the quality of the actual pattern which demonstrates that these proteins occur in larger amounts during the disease process and are not merely degradation products.

\section{References}

1. Felgenhauer, K. (1988) Liquordiagnostik. In: Labor and Diagnose (Thomas, L., ed.) pp. 1403-1446.

2. Reiber, H. (1983) Kinetics of protein agglomeration. A nephelometric method for the determination of total protein in biological samples. J. Biochem. Biophys. Methods $7,153-160$

3. Bollini, R. \& Chrispeels, M. J. (1978) Characterization and subcellular localization of vicilin and phytohemagglutinin, the two major reserve proteins of Phaseolus vulgaris $\mathrm{L}$. Planta 142, 291-298.

4. Secrest, J. P. \& Jackson, R. L. (1972) Molecular weight determination of glycoproteins by polyacrylamide gel electrophoresis in sodium dodecylsulfate. Methods in Enzymology $28,55-63$.

5. Mäder, M. \& Chrispeels, M. (1984) Synthesis of an integral protein of the protein body membrane in Phaseolus vulgaris. Planta $160,330-340$.

6. Bowen, B., Steinberg, J., Laemmli, U. K. \& Weintraub, H. (1980) The detection of DNA-binding proteins by protein blotting. Nucl. Acids Res. 8, 1-3.
It would be premature to draw any conclusions as to the biochemical nature and the pathophysiological meaning of these glycoproteins. Investigations are, however, in progress to elucidate both questions.

\section{Acknowledgement}

We thank Drs. W. Hösel and A. Haselbeck, Fa. Boehringer Mannheim, for helpful discussion and cooperation and Mrs $S$. Rudolph for introduction to isoelectric focusing.

7. Schipper, H. J., Kruse, H. \& Reiber, H. (1984) Silver staining of oligoclonal immunoglobulin $\mathrm{G}$ subfractions in cerebrospinal fluid after IEF in thin layer polyacrylamide gels. Science Tools 31, 5-6.

8. Haselbeck, A. \& Hösel, W. (1990) Description and application of an immunological detection system for analyzing glycoproteins on blots. Glycoconjugate J. 7, 63-74.

9. Haselbeck, A., Schickaneder, E., van der Elz, H., Hösel, W. (1990) Structural characterization of glycoprotein carbohydrate chains by using digoxigenin labeled lectins on blots. Anal. Biochem. 191, 25-30.

10. Schwick, H. G. \& Haupt, H. (1981) Purified human plasma proteins of unknown function. Japan. J. Med. Sci. Biol. 34, $299-327$.

11. Gries, A., Nimpf, J., Wurm, H., Kostner, G. M. \& Kenner, Th. (1989) Characterization of isoelectric subspecies of Asialo- $\beta 2$-glycoprotein I. Biochem. J. 260, 531-534.

Priv. Doz. Dr. M. Mäder

Neurologische Klinik der Universität

Robert Koch Str. 40

W-3400 Göttingen

Bundesrepublik Deutschland 
